\title{
Quand Msx1 permet aux myotubes de faire souche
}

La salamandre ne possède pas seulement le pouvoir - mythique - de traverser le feu impunément. Elle possède surtout celui, bien réel, de régénérer ses membres amputés, pattes ou queue. Cette propriété spectaculaire (dite régénération épimorphique) est associée à une aptitude à la réparation tissulaire plus générale. Ainsi, chez l'adulte, les cardiomyocytes peuvent entrer à nouveau dans le cycle cellulaire et participer à la restauration de la fonction cardiaque [1]. Pourquoi sommesnous, comme la totalité des vertébrés tétrapodes autres que les amphibiens urodèles, exclus du bénéfice de la régénération ? A défaut de répondre à cette question, un récent article de Cell [2] propose quelques pistes pour nous y réintégrer.

Chez les urodèles, la régénération d'un membre amputé commence par la formation d'une population de cellules indifférenciées sous l'épithélium cicatriciel, le blastème. Ces celIules proviennent, en partie, de la dédifférenciation de plusieurs tissus: muscle, derme, cartilage. Elles prolifèrent puis, progressivement, quittent le cycle cellulaire pour se différencier de nouveau, recréant tous les tissus du membre original de façon parfaitement ordonnée. Les cellules musculaires ont été particulièrement bien étudiées dans ce processus. Des myotubes introduits dans le blastème de régénération cessent d'exprimer les gènes caractéristiques de l'état différencié et, pour 15 à $20 \%$ d'entre eux, se fragmentent et donnent naissance à des cellules pluripotentes qui participent à la formation de nouveaux muscles et dans de rares cas, de cartilage $[1,3]$.

Le gène $M s \times 1$, qui code pour un facteur de transcription à homéoboîte, semble ne jamais être exprimé dans tère, a été proposé comme caractéristique de l'état indifférencié. De fait, dans l'embryon de souris, Msxl est exprimé dans une sous-population de myoblastes, mais ne l'est pas dans les cellules différenciées qui en dérivent [4]. De plus, son expression forcée dans des myoblastes en culture les maintient dans un état prolifératif et empêche leur différenciation [5]. Dans le blastème de régénération, la situation est plus confuse: une équipe y a observé une forte induction de Msx1 [6] tandis qu'une autre n'observe qu'une faible induction [7]. Faute de génétique, nul ne peut dire si cette induction est causale dans le processus de dédifférenciation, ou simplement coïncidente. Partant de ces observations, O delberg et al. [2] ont analysé l'effet de l'expression forcée de Msxl dans des myotubes différenciés de la lignée murine C2C12. L'expression forcée de Msx1 dans une population homogène de myotubes différenciés conduit une proportion notable d'entre eux (environ $9 \%$ ) à se fragmenter en myotubes plus petits, voire en cellules mononucléées qui prolifèrent. Si ces cellules dédifférenciées sont clonées et cultivées dans des conditions favorables à la différenciation vers un type cellulaire particulier (chondrogenèse, adipogenèse, ostéogenèse ou myogenèse), il se forme des foyers de cellules différenciées qui expriment les marqueurs caractéristiques du lignage choisi. Postulant que cette transdétermination est due à l'effet de Msxl, les auteurs forcent l'expression de ce gène directement dans les myoblastes indifférenciés de la lignée C2C12. Ces cellules acquièrent alors la capacité de s'engager dans différentes voies de différenciation, ce qui n'avait jamais été observé auparavant [5].

Ces observations provocantes soulèvent des questions importantes rela- tives à la plasticité de l'état différencié dans le lignage myogénique. On savait déjà que, quand on arrive à s'affranchir de la structure syncytiale des myotubes, les cellules musculaires différenciées, mononucléées, peuvent se dédifférencier [8]. La myoséverine, molécule issue de la chimie combinatoire, interagit avec les microtubules et conduit à la fragmentation des myotubes. Les cellules mononucléées ainsi produites entrent à nouveau dans le cycle cellulaire [9]. On sait par ailleurs que la protéine du rétinoblastome $\mathrm{Rb}$ joue un rôle critique dans le verrouillage antiprolifératif et la stabilité des fibres musculaires; un taux réduit de $\mathrm{Rb}$ chez des souris mutantes conduit à la formation de fibres musculaires dont les noyaux continuent à synthétiser I'ADN [10]. II faut noter aussi que les myoblastes de la lignée C2C12, utilisée comme modèle par $O$ delberg et al. sont intrinsèquement doués de pluripotence: ils peuvent par exemple se différencier en ostéocytes sous l'effet de la BMP2 (bone morphogenic protein) [11]. Aucun de ces travaux n'avait cependant abouti à l'obtention, à partir de myotubes, de cellules souches pluripotentes montrant un spectre de potentialités aussi large que celui qui est observé sous l'effet de Msxl. Savoir si c'est une des fonctions de Msxl que de maintenir un état de pluripotence dans les tissus où il s'exprime normalement est une question importante, qui devrait conduire à de nouvelles recherches sur ce gène.

Une des limites de cette approche pour la réparation tissulaire réside dans la maîtrise du délicat équilibre entre la reprise des cycles cellulaires et la nécessité impérieuse de limiter la prolifération cellulaire, sous peine de former des cellules tumorales. L'expression forcée de Msxl dans les 
myoblastes a aussi pour effet de les transformer [5]. De ce point de vue, nous avons encore à apprendre des urodèles: les cellules du blastème partagent avec les cellules souches leur capacité à proliférer indéfiniment (au moins 200 cycles) sans montrer de signes de crise ou de sénescence, et cependant, ces cellules sont remarquablement résistantes à la transformation par des carcinogènes chimiques. Peut-être ces propriétés sont-elles liées à l'activité de quelques gènes, dont $M s x 1$, qu'il suffirait de réintroduire - ou de réactiver - dans nos cellules. Mais plusieurs données suggèrent plutôt que les modalités de régulation de la prolifération, de la différenciation, de la sénescence et de la néoplasie sont sensiblement différentes entre les cellules d'urodèles et de mammifères. Ainsi, les noyaux des myotubes différenciés des urodèles peuvent entrer en cycle sans dommage, au contraire de ceux des mammifères [12]. II faudra sans doute mieux définir les propriétés des cellules du blastème avant de savoir si elles peuvent être transposées à la cellule humaine, et de déterminer la place d'une approche fondée sur l'expression forcée de Msxl dans l'arsenal des stratégies actuellement élaborées pour la réparation tissulaire. Cependant, si I'on considère qu'une des vertus cardinales d'une cellule souche est de demeurer quiescente sur de longues périodes, pourquoi une cellule différenciée comme le myotube, dans laquelle le cycle cellulaire est bloqué, ne pourrait-elle pas constituer un réservoir de cellules souches?

1. Brockes JP. Amphibian limb regeneration: rebuilding a complex structure. Science 1997; 276 : 81-7.

2. Odelberg SJ, Kollhoff A, Keating MT. Dedifferentiation of mammalian myotubes induced by msx1. Cell 2000; 103: 1099-109.

3. Kumar A, Velloso CP, Imokawa Y, Brockes JP. Plasticity of retrovirus-labelled myotubes in the newt limb regeneration blastema. Dev Biol 2000; 218: 125-36.

4. H ouzelstein D, Auda-Boucher G, Cheraud Y, et al. The homeobox gene Msxl is expressed in a subset of somites, and in muscle progenitor cells migrating into the forelimb. Development 1999; 126: 2689-701.

5. Song $K$, Wang $Y$, Sassoon D. Expression of Hox7.1 in myoblasts inhibits terminal differentiation and induces cell transformation. Nature 1992 ; $360: 477-81$.
6. Simon HG, Nelson C, Goff D, et al. Differential expression of myogenic regulatory genes and Msx-1 during dedifferentiation and redifferentiation of regenerating amphibian limbs. Dev Dyn 1995; 202: 1-12.

7. Crews L, Gates PB, Brown R, et al. Expression and activity of the newt Msx-1 gene in relation to limb regeneration. Proc Roy Soc L ondon B Biol Sci 1995; 259: 161-71.

8. Devlin $\mathrm{BH}$, Konigsberg IR. Reentry into the cell cycle of differentiated skeletal myocytes. Dev Biol 1983; 95: 175-92

9. Rosania GR, Chang YT, Perez O, et al. Myoseverin, a microtubule-binding molecule with novel cellular effects. Nat Biotech $2000 ; 18$ : 304-8.

10. Zacksenhaus $E$, Jiang $Z$, Chung $D$, et al. $p R b$ controls proliferation, differentiation, and death of skeletal muscle cells and other lineages during embryogenesis. Genes Dev 1996; 10: 3051-64.

11. Nishimura R, Kato, $Y$ Chen, D et al. Smad5 and DPC4 are key molecules in mediating BMP-2induced osteoblastic differentiation of the pluripotent mesenchymal precursor cell line C2C12. J Biol Chem 1998; 273 : 1872-9.

12. Tanaka EM, Gann AA, Gates PB, Brockes JP. Newt myotubes reenter the cell cycle by phosphorylation of the retinoblastoma protein. J Cell Biol 1997; 136: 155-65.

\section{Benoît Robert Didier Montarras}

Institut Pasteur, 25, rue du DocteurRoux, 75724 Paris Cedex 15, France.

\section{BRÈVES}

11 couleurs, 13 paramètres $=$ 75 cellules... Le fonctionnement «normal » du système immunitaire dépend de la présence efficace de différentes sous-populations Iymphocytaires, chacune devant être présente en nombre strictement contrôlé. Toute perturbation dans cet équilibre délicat peut refléter la pathogénie d'une maladie plus ou moins grave (anémie, cancer, SIDA). Les modifications quantitatives dans les sous-populations lymphoïdes principales sont classiquement évaluées par des analyses en cytométrie de flux. En routine, il est cependant difficile d'analyser une population de cellules selon plus de trois paramètres à la fois, en raison du nombre limité de fluorochromes ayant des spectres suffisamment distincts. Ces limitations sont-elles en passe de devenir obsolètes? Un groupe du NIH rapporte la possibi- lité de combiner désormais 11 paramètres de fluorescence différents [1] . Les résultats obtenus par cette approche, appelée PFC (polychromatic flow cytometry), ont été comparés avec une analyse fonctionnelle (mesure de la sécrétion d'IFN $\gamma$, également en fluorescence), et celle du répertoire du récepteur des populations $T$ naïves et mémoires. La correspondance entre les résultats obtenus par ces différentes approches est parfaite, alors que les analyses classiques de fluorescence limitées à 3 couleurs fournissent des résultats discordants, dus à l'inclusion erronée d'un faible pourcentage de cellules T mémoires dans la population des cellules naïves. Or, la quantification précise de ces deux populations peut revêtir une grande importance dans le contexte d'infections chroniques ou aiguës. Ainsi, dans le cas des patients HIV+, l'effondrement des taux de lymphocytes naïfs $C D 4^{+}$et $C D 8^{+}$suit l'évolution de la maladie, et la reconstitution immunitaire apportée par les traitements antiviraux doit donc être évaluée avec un soin particulier. Lorsqu'elle est optimisée, la technique de PFC permet d'identifier jusqu'à 75 sous-populations différentes, susceptibles d'exercer des fonctions distinctes. L'énumération de ces populations pourrait se révéler utile sur le plan clinique, qu'il s'agisse de corréler la quantification de sous-populations distinctes avec des états pathologiques ou de réaliser une analyse fonctionnelle sur des cellules extrêmement peu représentées.

[1. De Rosa SC, et al. Nat Biotech $2001 ; 7: 245-8]$ 